Abstract

\title{
Self-Heated Graphene Microchannels for Low-Power-Consumption Chemoresistive Sensor Array ${ }^{\dagger}$
}

\author{
Ho Won Jang ${ }^{1, *}$ and Soo Young Kim ${ }^{2}$ \\ 1 Department of Materials Science and Engineering, Seoul National University, Seoul 08826, Korea \\ 2 School of Chemical Engineering and Materials Science, Chung-Ang University, Seoul 06974, Korea; \\ sooyoungkim@cau.ac.kr \\ * Correspondence: hwjang@snu.ac.kr \\ + Presented at the 8th GOSPEL Workshop. Gas Sensors Based on Semiconducting Metal Oxides: Basic \\ Understanding \& Application Fields, Ferrara, Italy, 20-21 June 2019.
}

Published: 19 June 2019

The Internet of Everything (IoE) refers to billions of objects having intelligent connections with processed data. Sensors hold the key to the IoE as they detect and assess the internal and external states of objects. In particular, gas sensors contribute greatly to make people's lives better via transmitting information about gas species in ambient air. 2D materials such as graphene and transition metal disulfides are being extensively studied for gas-sensing applications due to their high sensitivity at room temperature, transparency, and flexibility. However, they have drawbacks such as low selectivity and irreversible sensing behaviors. To overcome these drawbacks, the chemoresistive sensing properties of the $2 \mathrm{D}$ materials have been modified by diverse approaches such as introducing extrinsic defects, functionalization, and noble metal decoration. Here, we present unprecedented gas detection using self-activated graphene microchannels with surface decoration. Joule heating by applying voltages in graphene microchannels is very effective to make the materials operate at elevated temperatures and enhance gas sensing properties such as response and recovery. Compared to pristine graphene sensors, the noble-metal-decorated graphene sensors exhibit highly improved gas-sensing properties upon exposure to various gases (Figure 1). In particular, an unexpected substantial enhancement in $\mathrm{H}_{2}$ detection is found, which has never been reported for $\mathrm{Au}$ decoration on any types of chemoresistive materials. We show $2 \times 2$ gas sensors array based on metal-decorated graphene microchannels that operate with low power consumption without additional heaters. The discovery of such a new functionality in the existing material platform holds the key to diverse research areas based on metal nanoparticles/2D material heterostructures. 

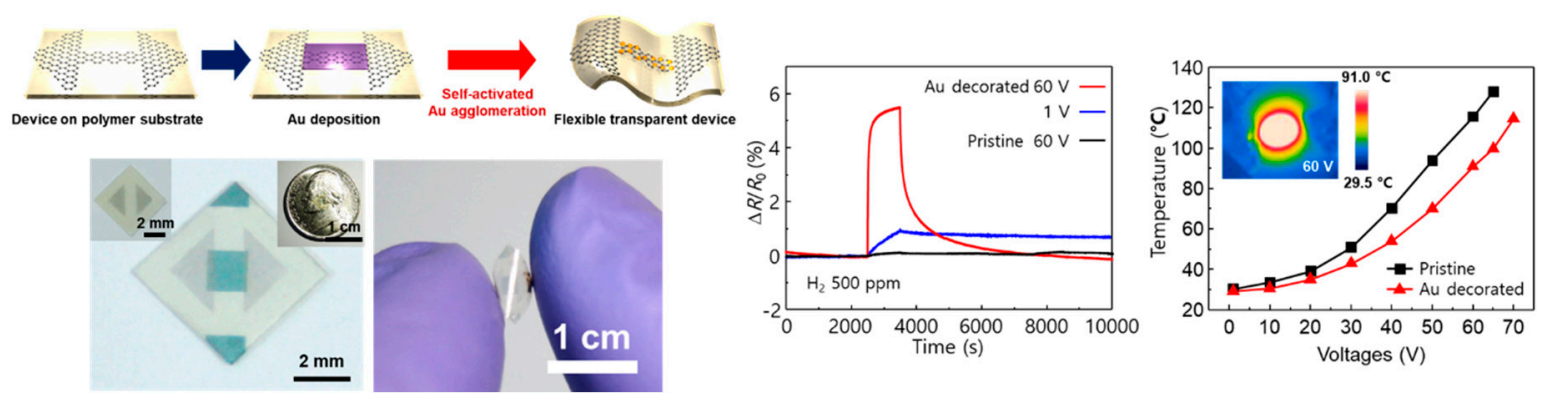

Figure 1. Transparent and flexible Au-decorated graphene microchannel gas sensor (Ref: Kim et al., Nanoscale, 2019, 11, 2966-2973).

C 2019 by the authors. Licensee MDPI, Basel, Switzerland. This article is an open access article distributed under the terms and conditions of the Creative Commons Attribution (CC BY) license (http://creativecommons.org/licenses/by/4.0/). 